\title{
The potential role of Gliptins to fight COVID-19
}

\author{
Francesco Ferrara ${ }^{1}$ and Antonio Vitiello ${ }^{1}$ \\ ${ }^{1}$ Azienda Unità Sanitaria Locale Umbria 1
}

July 16, 2020

\begin{abstract}
People with diabetes represent a population at greater risk of infection and complications from Sars-Cov-2 (COVID-19), Diabetes represents one of the most important comorbidities related to the severity of viral infection causing an increased risk of serious complications such as severe acute respiratory syndrome and multi-organ dysfunction associated with a hyperinflammatory state. Glycemic normalization in patients with diabetes must be managed in the best possible way even during COVID-19 infection to avoid serious complications. However, for some antidiabetic agents such as DPP-4 inhibitors (gliptins) there is evidence of efficacy against COVID-19 extra pancreatic glycemic normalization. The objective of this article is to provide an overview of current evidence on the potential therapeutic benefits of gliptins to combat Sars-Cov-2 infection.
\end{abstract}

\section{Introduction}

\section{COVID-19 infection and diabetes}

The global pandemic caused by the new Sars-Cov-2 (COVID-19) began in China in November 2019 and quickly became a global problem, representing a health emergency. To date, about 6.15 million positive cases and 372,000 deaths have been recorded with over 260 countries affected (1). COVID-19 infection can range from asymptomatic or slightly symptomatic to more severe stages such as multi-organ dysfunction resulting in fatal lung injury and thrombus formation. Studies show that people with pre-existing chronic diseases such as diabetes are at increased risk of infection and serious complications. To date, there are no direct antivirals and effective vaccines against Sars-Cov-2 (2). During the current global COVID-19 pandemic, diabetes has been identified as an important risk factor for the development of severe Sars-Cov-2 viral pneumonia (3).

Epidemiological data indicate that the risk of a fatal outcome caused by COVID-19 infection is up to $50 \%$ higher in patients with diabetes (4). There are many factors that may increase the risk of serious complications for the diabetic patient with COVID-19 such as the presence of a deficient immune system, an inadequate fibrinolytic cascade, an overactive inflammatory state, and secondary cardiovascular disease caused by diabetes.(5). In addition, it should be considered that the Sars-Cov-2 virus uses the angiotensin 2 conversion enzyme protein (ACE2) to penetrate the epithelial cells of the lung. ACE2 has a protective role especially with regard to inflammation of the respiratory tract. COVID-19 infection in the most severe stages reduces the expression of ACE2, this is one of the causes of hyperinflammation and respiratory failure (6-7), moreover diabetes also changes the expression of ACE2, and this can cause an even more complicated and serious clinical situation. Finally, the viral infection itself can cause a worsening of the diabetes disease with a more difficult glycaemia to control (8). A fundamental aspect to highlight is that the DPP-4 protein (therapeutic target of glyptins) has been identified as a functional receptor for the virus responsible for MERS (9), a virus similar to that of COVID-19, to date it is not certain that it is also a functional receptor for Sars-Cov-2, investigations in this direction are underway. Moreover, diabetes patients have an altered expression of the DPP-4 protein, this factor may contribute to overactive inflammation, which could further complicate the clinical situation. Based on the considerations expressed, it emerges that the management 
of the patient with diabetes and COVID-19 positive is extremely delicate. The recommendations indicate that drug treatment for diabetes should not be discontinued during viral infection if there are no particular contraindications, however for some antidiabetes drugs such as glyptins there is evidence in the literature showing their potential additional therapeutic role against COVID-19 infection.

\section{Gliptins agents and potential efficacy against COVID-19}

Gliptins (sitagliptin, vildagliptin, etc..) are DPP-4 inhibitors with undoubted therapeutic efficacy in the anti-diabetes field. Recently for this class of drugs evidence has shown extra pancreatic pleiotropic activity of glycemic normalization. The DPP-4 protein has several actions, in particular it plays an important role in the regulation of the immune system by activating T cells and regulating CD86 expression, and is responsible for increasing inflammation in patients with diabetes. It should also be noted that the activity of DPP-4 can influence the function of several cytokines, chemokines and growth factors. Some studies have suggested that a higher mortality rate and complications in people with diabetes and MERS infected may be associated with a dysregulated immune response mediated by the DPP-4 protein (10-11), so a potential therapeutic role of glyptins in fighting COVID-19 infection is also to be assumed. However, to date, the effects of DPP-4 inhibition on the immune system and inflammation are still not fully understood.

A meta-analysis has shown that upper respiratory tract infections do not increase significantly with inhibitory treatment of DPP-4 (12), moreover MERS-CoV uses DPP-4 to enter host cells (13), but it is not known whether Sars-Cov-2 uses the same protein to enter the cell as well, in addition to ACE-2, If this were demonstrated, the use of glyptin could decrease the risk of Sars-Cov-2 infection, but for now it is only a hypothesis. The potential benefit in the treatment of Sars-Cov-2 infection with DPP-IV inhibitors remains to be investigated. To date, it is not entirely clear whether DPP-IV inhibition may play an important role in controlling inflammation in patients with diabetes and COVID-19, but it may be a potential target to prevent and reduce the risk and progression of acute airway complications that a patient with diabetes may have in addition to COVID-19 infection (14).

\section{Conclusions}

The COVID-19 global pandemic represents one of the greatest health challenges in the history of mankind. Patients with comorbidities such as diabetes may be at greater risk of complications if infected with COVID19. Treatment of diabetes in a patient with COVID-19 must be carefully managed, for some anti diabetes agents such as gliptins there is evidence of extra pancreatic pleiotropic effects and glycemic normalization, which could be an added value in the fight against COVID-19 infection in the patient with diabetes.

\section{Conflicts of interest}

None of the Authors have conflicts of interest to disclose.

\section{Founds}

None

\section{Copyright}

The authors certify that the manuscript is original, never submitted to other journal for publication before. All authors contributed equally to the manuscript and had the opportunity to revise and approve the final text.

\section{Disclosure statement:}

Dr. A.Vitiello has nothing to disclose

Dr. F.Ferrara has nothing to disclose

\section{References}


1)World Health Organization HO (2020) Coronavirus disease 2019 (COVID-19) situation report Available fromhttps://www.who.int/emergencies/diseases/novel-coronavirus-2019/situation-reports.

2)Lin L, Lu L, et al. (2020) Hypothesis for potential pathogenesis of Sars-CoV-2 infection a review of immune changes in patients with viral pneumonia. Emerg Microbes Infect.

3)Zou Q, Zheng S et al. Influenza A-associated severe pneumonia in hospitalized patients: risk factors and NAI treatments. Int J Infect Dis 2020.

4)Remuzzi A, Remuzzi G. COVID-19 and Italy: what next? Lancet 2020.

5)Stefan R Bornstein et al. Practical recommendations for the management of diabetes in patients with COVID-19 Lancet Diabetes Endocrinol 2020.

6)Hoffmann M, Kleine-Weber $\mathrm{H}$ et al. SARS-CoV-2 cell entry depends on ACE2 and TMPRSS2 and is blocked by a clinically proven protease inhibitor. Cell 2020 .

7)Bindom SM, Lazartigues E. The sweeter side of ACE2: physiological evidence for a role in diabetes. Mol Cell Endocrinol 2009.

8)Yang JK, Lin SS et al. Binding of SARS coronavirus to its receptor damages islets and causes acute diabetes. Acta Diabetol 2010.

9)Raj VS, Mou H et al. Dipeptidyl peptidase 4 is a functional receptor for the emerging human coronavirusEMC. Nature 2013.

10)Li K, Wohlford-Lenane CL, Channappanavar R et al. Mouse-adapted MERS coronavirus causes lethal lung disease in human DPP4 knockin mice. Proc Natl Acad Sci USA 2017.

11)Fan C, Wu X et al. A Human DPP4- Knockin Mouse's Susceptibility to Infection by Authentic and Pseudotyped MERS-CoV. Viruses 2018.

12)Yang W, Cai X et al. DPP-4 inhibitors and risk of infections: a meta-analysis of randomized controlled trials.

Diabetes Metab Res Rev 2016.

13)Raj VS, Mou H et al. Dipeptidyl peptidase 4 is a functional receptor for the emerging human coronavirusEMC. Nature (2013)

14)Iacobellis G. COVID-19 and diabetes: can DPP4 inhibition play a role? DiabetesResClinPract (2020). 